\title{
Energy conversion through mass loading of escaping ionospheric ions for different Kp values
}

\author{
Masatoshi Yamauchi ${ }^{1}$ and Rikard Slapak ${ }^{2}$ \\ ${ }^{1}$ Swedish Institute of Space Physics (IRF), P.O. Box 812, 98128 Kiruna, Sweden \\ ${ }^{2}$ Division of Space Technology, Luleå University of Technology, Kiruna, Sweden \\ Correspondence: Masatoshi Yamauchi (m.yamauchi@irf.se)
}

Received: 7 July 2017 - Revised: 22 November 2017 - Accepted: 23 November 2017 - Published: 4 January 2018

\begin{abstract}
By conserving momentum during the mixing of fast solar wind flow and slow planetary ion flow in an inelastic way, mass loading converts kinetic energy to other forms - e.g. first to electrical energy through charge separation and then to thermal energy (randomness) through gyromotion of the newly born cold ions for the comet and Mars cases. Here, we consider the Earth's exterior cusp and plasma mantle, where the ionospheric origin escaping ions with finite temperatures are loaded into the decelerated solar wind flow. Due to direct connectivity to the ionosphere through the geomagnetic field, a large part of this electrical energy is consumed to maintain field-aligned currents (FACs) toward the ionosphere, in a similar manner as the solar wind-driven ionospheric convection in the open geomagnetic field region. We show that the energy extraction rate by the mass loading of escaping ions $(\Delta K)$ is sufficient to explain the cusp FACs, and that $\Delta K$ depends only on the solar wind velocity accessing the mass-loading region $\left(u_{\mathrm{sw}}\right)$ and the total mass flux of the escaping ions into this region $\left(m_{\text {load }} F_{\text {load }}\right)$, as $\Delta K \sim-m_{\text {load }} F_{\text {load }} u_{\text {sw }}^{2} / 4$. The expected distribution of the separated charges by this process also predicts the observed flowing directions of the cusp FACs for different interplanetary magnetic field (IMF) orientations if we include the deflection of the solar wind flow directions in the exterior cusp. Using empirical relations of $u_{0} \propto \mathrm{Kp}+1.2$ and $F_{\text {load }} \propto \exp (0.45 \mathrm{Kp})$ for $\mathrm{Kp}=1-7$, where $u_{0}$ is the solar wind velocity upstream of the bow shock, $\Delta K$ becomes a simple function of $\mathrm{Kp}$ as $\log _{10}(\Delta K)=0.2 \cdot \mathrm{Kp}+$ $2 \cdot \log _{10}(\mathrm{Kp}+1.2)+$ constant. The major contribution of this nearly linear increase is the $F_{\text {load }}$ term, i.e. positive feedback between the increase of ion escaping rate $F_{\text {load }}$ through the increased energy consumption in the ionosphere for high $\mathrm{Kp}$, and subsequent extraction of more kinetic energy $\Delta K$ from
\end{abstract}

the solar wind to the current system by the increased $F_{\text {load }}$. Since $F_{\text {load }}$ significantly increases for increased flux of extreme ultraviolet (EUV) radiation, high EUV flux may significantly enhance this positive feedback. Therefore, the ion escape rate and the energy extraction by mass loading during ancient Earth, when the Sun is believed to have emitted much higher EUV flux than at present, could have been even higher than the currently available highest values based on $\mathrm{Kp}=9$. This raises a possibility that the ion escape has substantially contributed to the evolution of the Earth's atmosphere.

Keywords. Magnetospheric physics (magnetosphereionosphere interactions)

\section{Introduction}

The effect of mass loading has long been discussed in relation to solar wind interaction with comets (Biermann et al., 1967; Kömle and Lichtenegger, 1984; Szegö et al., 2000, and references therein) and unmagnetized bodies, such as Venus and Mars (Breus et al., 1989; Szegö et al., 2000, and references therein). This effect was originally considered for newly born cold ions in cometary environments and planetary exospheres, where cometary/exospheric atoms are exposed to the solar wind before they are ionized with negligible initial velocity as compared to the solar wind velocity in the planetary frame. In these applications, the mass-loading effect transfers momentum and energy from the solar wind to the newly born ions by driving these ions to move with finite velocities along cycloid trajectories in the solar wind electric field and interplanetary magnetic field (IMF).

The general concept of mass loading can be applied to any mixture of plasmas with different velocities if the slower 
component (e.g. planetary ions) gains momentum by experiencing the motional electric field $\boldsymbol{E}=-\boldsymbol{u} \times \boldsymbol{B}$ of the faster component, where $\boldsymbol{u}$ is the velocity (e.g. the local solar wind velocity) and $\boldsymbol{B}$ is the local magnetic field (e.g. IMF). The slower component does not have to be cold ions because the $\boldsymbol{E} \times \boldsymbol{B}$ convection velocity will be added even to heated ions. Also, the momentum transfer does not have to be localized (e.g. can be remote when it is connected by sufficiently strong magnetic fields) as long as the motional electric field accelerates the slower component as bulk plasma (Alfvén and Fälthammar, 1963, Sects. 3.2.1 and 3.11.1). For example, the ionospheric plasma gaining anti-sunward velocity in the open geomagnetic field region (Dungey, 1961) through the dynamo-motor mechanisms connected by the geomagnetic field (Akasofu, 1975) can also be considered as a mass-loading plasma to the solar wind flow on the same geomagnetic field lines. As a consequence, the anti-sunward solar wind in the magnetospheric boundary (e.g. across the polar magnetopause for southward IMF) is expected to lose its anti-sunward momentum by the $\boldsymbol{J} \times \boldsymbol{B}$ force, where $\boldsymbol{J}$ is the electric current density (in the present case, the magnetopause Chapman-Ferraro current) flowing against $\boldsymbol{E}$ (Roederer, 1977). The source of the motional electric field is not limited to the open magnetic field - viscous-like interaction at the magnetospheric boundary (Axford and Hines, 1961), e.g. through Kelvin-Helmholtz instability (Hasegawa et al., 2004), can also provide such an electric field in the magnetosphere.

In a first-order approximation of momentum transfer from the solar wind to the ionosphere, the mass (or inertia) of the magnetospheric plasma is normally ignored compared to that of the ionospheric plasma above the $\mathrm{E}$ region. However, ion data from Cluster and Polar have revealed that a large amount of ionospheric ions are kept supplied to the exterior cusp, plasma mantle and magnetosheath, with a total flux of about $10^{24-26} \mathrm{~s}^{-1}$ (Peterson et al., 2008; Nilsson, 2011; Slapak et al., 2013, 2015, 2017) and with a local number density ratio to the solar wind of about $1 \%$. This amount is sufficient to substantially decelerate the solar wind by about $10 \%$ because of 16 times heavier mass of $\mathrm{O}^{+}$than $\mathrm{H}^{+}$, and might significantly influence the electrodynamics of the polar ionosphere, e.g. by the formation of a localized independent current system in the cusp region (Yamauchi and Slapak, 2017).

Since the spatial distribution of the escaping ions above the ionosphere is very localized near the cusp and auroral zone (e.g. Peterson et al., 2008), the mass-loading effect should also be localized and completely independent of the massloading effect of the ionospheric $\mathrm{E}$ region mentioned above. Therefore, this effect must be considered differently than the traditional solar wind-magnetosphere-ionosphere coupling. Although localized to the cusp region at the ionospheric altitude, this region is mapped to a large area at high altitudes with a scale size much larger than the ion gyroradius (in the plasma mantle, the geomagnetic field strength is about 25$30 \mathrm{nT}$ where the ion flux of escaping ions is maximized (Sla- pak et al., 2017), and the gyroradius is $<180 \mathrm{~km}$ for $1 \mathrm{keV}$ $\mathrm{H}^{+}$and $<1300 \mathrm{~km}$ for $3 \mathrm{keV} \mathrm{O}^{+}$). Therefore, we can assume in the model that the mass loading will be completed within the plasma mantle.

Due to a strong dependence on Kp and solar EUV of the ion outflow rate in the cusp and its vicinity (Norqvist et al., 1996; Cully et al., 2003; Peterson et al., 2006; Slapak et al., 2017), the expected current system should have a stronger dependence on Kp and EUV than what the current system relevant to the global electrodynamics has (e.g. Fujii and Iijima, 1987; Thomsen, 2004, and references therein). One obvious candidate for this current system is the cusp current system (Potemra, 1994; Yamauchi and Slapak, 2017, and references therein), which is composed of one or two pairs of cusp field-aligned currents (FACs) flowing mainly inside the cusp (equatorward and poleward) and the ionospheric currents closing the current circuit with these FACs.

Many different terminologies have been used for the FAC part of the cusp current system, as summarized in Yamauchi and Slapak (2017), and we here use the terminology of cusp Region 1 FAC and cusp Region 0 FAC for the equatorward part and the poleward part of the cusp FACs, respectively. Cusp Region 1 FAC and cusp Region 0 FAC are located approximately at the extended location of the dayside (noncusp) Region 1 FAC and the Region 0 FAC toward midday, respectively (Iijima and Potemra, 1976; Heikkila, 1984; Yamauchi et al., 1993b; Potemra, 1994), while multi-spacecraft satellite observations at low altitudes demonstrated that they are independent of each other in terms of locations (e.g. Ohtani et al., 1995; for review, Yamauchi and Slapak, 2017). Here, the dayside (non-cusp) Region 1 FAC is the strongest persistent FAC flowing into the ionosphere in the dawn sector and flowing out from the ionosphere in the afternoon sector in both hemispheres (Iijima and Potemra, 1976; Potemra, 1994).

To develop the scenario of explaining the cusp FACs by energy conversion (dynamo) through mass loading, it is important to examine the distribution and strength of the expected current system by such a "mass-loading dynamo" for different IMF directions, different $\mathrm{Kp}$, and different solar wind parameters. Among these parameters, only the Kp dependence can be estimated from the statistics of ion outflow, because the solar wind and IMF dependences on the outflow in the exterior cusp and plasma mantle are not well understood (only the Kp dependence has been obtained). Considering these limitations, this paper makes a general formulation for this mass-loading dynamo, and estimates its dependence on $\mathrm{Kp}$ and the relevant current system distribution for different IMF orientations.

\section{Energy conversion by mass loading}

The deceleration of fast plasma inflow (flowing in the $-x$ direction, e.g. the solar wind) with speed $u$ and mass density $\rho$, 
after full mass loading with newly born planetary/cometary heavy ions that have zero bulk velocity (i.e. the bulk velocities of both species become the same), is described by the conservation of momentum flux:

$(\rho+\Delta \rho) \cdot(u+\Delta u)^{2}=\rho u^{2}$,

where $\Delta \rho$ is the mass density of the mass-loading ions, and $u+\Delta u$ is the final speed after full mass loading. This relation is valid even when mass-loading ions are introduced into the system from a different direction than $x$ (we call this direction as $z$ ), e.g. along the geomagnetic field for the Earth's case as long as the bulk motion in the $z$ direction is not affected. Here, small letters $x, y$ and $z$ represent general coordinates used for ideal mathematical formulation. Large letters $X Y$, and $Z$ that appear later (e.g. in Fig. 1) represent the global coordinates in the actual Sun-Earth geometry, and the Greek letters $\xi$ and $\eta$ (e.g. Fig. 3) represent local coordinates of the actual flow in the exterior cusp, i.e. $\xi \sim x$ and $\eta \sim y$. Note that the mass flux, which has the dimension of momentum per unit volume, is not conserved, due to addition of $\Delta \rho$ into the system as either newly born ions (planetary/cometary cases) or through the $z$ direction (Earth's case).

After full mass loading, the total kinetic energy flux (we use the terminology "kinetic power" hereafter to avoid confusion) flowing through a surface $S$ perpendicular to the $x$ direction changes from $K=\rho u^{3} S / 2$ to $K+\Delta K=(\rho+$ $\Delta \rho)(u+\Delta u)^{3} S / 2$, with a relation of

$$
\begin{aligned}
\frac{K+\Delta K}{K} & =\left(\frac{u+\Delta u}{u}\right) \cdot \frac{(\rho+\Delta \rho) \cdot(u+\Delta u)^{2}}{\rho u^{2}} \\
& =\left(\frac{\rho}{\rho+\Delta \rho}\right)^{1 / 2},
\end{aligned}
$$

where we have used Eq. (1), or

$$
\frac{\Delta K}{K}=\left(1+\frac{\Delta \rho}{\rho}\right)^{-1 / 2}-1
$$

For very small $\Delta \rho \rightarrow d \rho$, this becomes

$$
\frac{\mathrm{d} K}{K} \approx-\frac{\mathrm{d} \rho}{2 \rho} .
$$

If the planetary ions are kept supplied by new ionization of the exospheric neutrals (Mars and Venus) or outgassing neutrals (comet), we simply replace $\rho$ and $\Delta \rho$ by $m_{\mathrm{sw}} n_{\mathrm{sw}}$ and $m_{\text {load }} p_{\text {load }} \tau$ in Eq. (2), where $m_{\text {sw }}$ and $m_{\text {load }}$ are the average masses of the fast inflow and newly produced mass-loading ions, respectively, $n_{\mathrm{sw}}$ is the number density of the fast inflow, and $\tau$ is the duration that the inflow spends travelling through the ion production region with mass-loading ions in which the volume ion production rate is $p_{\text {load }}$. By assuming $\mathrm{O}^{+}$for the mass-loading ions and $\mathrm{H}^{+}$for incident flow, we have

$$
\frac{\Delta K}{K}=\left(1+16 \cdot \frac{p_{\text {load }} \tau}{n_{\mathrm{sw}}}\right)^{-1 / 2}-1
$$

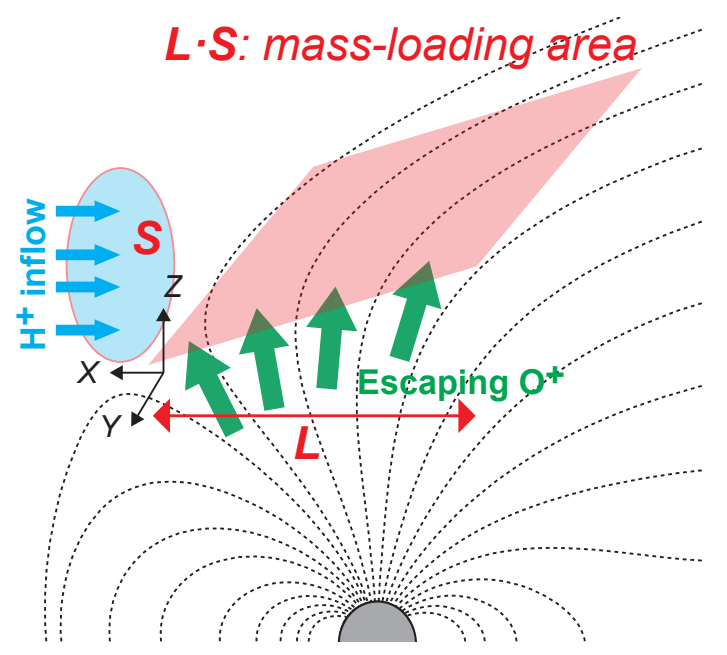

Figure 1. The mass-loading region that we consider (red). Solar wind $\mathrm{H}^{+}$ions (blue arrows) access this region through the blue area (cross section $S$ ), with a width of about $4-10 R_{\mathrm{E}}$ in the $Y$ direction and a thickness of about $5-6 R_{\mathrm{E}}$ in the $Z$ direction (although there is no solid observational statistics for the plasma mantle, these are good estimates, and these numbers disappear from the final formula anyway). The extent of the region in the anti-flow direction $(-X$ direction) is $L$.

The converted energy is used first to azimuthally accelerate $\mathrm{O}^{+}$along the motional electric field, which is simultaneously subject to the Lorentz force proportional to the speed, to make cycloid motion. If $p_{\text {load }}$ is significantly high, the azimuthal acceleration causes detectable azimuthal bulk deflection of the incident $\mathrm{H}^{+}$flow as observed in the comet environment of 67P/Churyumov-Gerasimenko by the Rosetta Plasma Consortium Ion Composition Analyser (RPC-ICA), which showed that such a deflection is even more drastic than expected, whatever the heliocentric distance (Behar et al., 2016, 2017).

\subsection{Amount of converted kinetic energy by the escaping $\mathrm{O}^{+}$}

Instead of ionization, we here consider escaping ionospheric ions flowing into the terrestrial exterior cusp and plasma mantle as shown in Fig. 1. The largest differences from the new ionization (cometary) case are as follows: (a) the massloading regions are geomagnetically connected to the ionosphere through which separated charges can flow, and (b) the mass-loading ions are already heated with non-zero gyrating velocity when they are supplied from one boundary along the magnetic field perpendicular (in the $z$ direction) to the inflow. In Cluster observations at the Earth, these mass-loading ions coming from the ionosphere have thermal velocities of $>100 \mathrm{~km} \mathrm{~s}^{-1}$ in Nilsson et al. (2006) and Waara et al. (2011). This value is comparable to the decelerated solar wind speed (about $100 \mathrm{~km} \mathrm{~s}^{-1}$ in the plasma mantle) as shown in Fig. 2, in which the bulk velocities of $\mathrm{O}^{+}$and $\mathrm{H}^{+}$along the average 


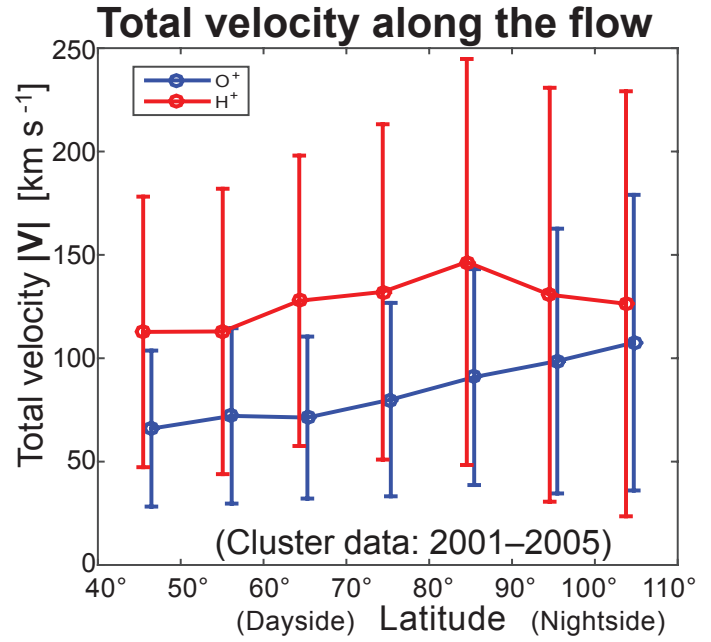

Figure 2. Bulk velocity profiles of $\mathrm{H}^{+}$(red) and $\mathrm{O}^{+}$(blue) along the average $\mathrm{O}^{+}$flow direction over plasma mantle as defined by Fig. 6 of Slapak et al. (2017) and obtained from the same statistics (Cluster, 2001-2005). Error bars defined by the standard deviation are given.

$\mathrm{O}^{+}$stream line in the plasma mantle (as defined in Slapak et al., 2017) are plotted. Fig. 2 further showed that $\mathrm{H}^{+}$is decelerated whereas $\mathrm{O}^{+}$is accelerated along a region which is nearly parallel to the $\mathrm{O}^{+}$outflow. Such velocity profiles of $\mathrm{O}^{+}$and $\mathrm{H}^{+}$in the plasma mantle are consistent with mass loading, i.e. momentum transfer from the solar wind $\mathrm{H}^{+}$to the escaping $\mathrm{O}^{+}$. Liao et al. (2015) also showed extra $\mathrm{O}^{+}$ acceleration between the cusp and nightside magnetosphere other than the centrifugal acceleration (Cladis, 1986; Nilsson et al., 2008).

We first estimate the energy conversion rate using the density ratio in a similar way as Yamauchi and Slapak (2017). For simplicity, we ignore the $-x$ component of the bulk velocity of the mass-loading $\mathrm{O}^{+}$at the boundary. This means that the mass loading effect mainly adds anti-sunward bulk velocity $(\boldsymbol{E} \times \boldsymbol{B}$ convection velocity) but not very much gyrovelocity to the mass-loading ions. We also assume values of parameters as follows: the local solar wind density and velocity just before accessing the mass-loading region are $n_{\mathrm{sw}}=5 \mathrm{~cm}^{-3}, u_{\mathrm{sw}}=200 \mathrm{~km} \mathrm{~s}^{-1}$, respectively, and the cross section of the solar wind access to the mass-loading region (see Fig. 1) is $S=20-60 R_{\mathrm{E}}^{2}=1-3 \times 10^{9} \mathrm{~km}^{2}$, where $R_{\mathrm{E}}$ is the Earth's radius ( $S$ depends strongly on the solar wind and IMF condition, but disappears from the final formula later). The total (number) flux and power of the solar wind inflow accessing this mass-loading region are estimated as

$$
\begin{aligned}
& n_{\mathrm{sw}} u_{\mathrm{sw}} S=1-3 \times 10^{27} \mathrm{~s}^{-1} \text { and } \\
& K_{\mathrm{in}}=\frac{m_{p} n_{\mathrm{sw}} u_{\mathrm{sw}}^{3} S}{2} \\
& =3-10 \times 10^{10} \mathrm{~W} .
\end{aligned}
$$

The estimated values of the number flux indicate that the escaping flux of $\mathrm{O}^{+}$into this mass-loading region is 1$2 \times 10^{25} \mathrm{~s}^{-1}$ on average (Nilsson, 2011; Slapak et al., 2017), which is around $1 \%$ of the incident solar wind flux $(\mathrm{d} \rho / \rho$ about $10-20 \%$ after multiplying with the mass ratio of 16 ). Therefore, we expect 5-10\% conversion rate from the kinetic power of the solar wind according to Eq. (3), i.e. $\Delta K=0.1-1 \times 10^{10} \mathrm{~W}$, into other energy forms.

To properly treat the dependence on the parameters at both boundaries (for the solar wind and ion escape), we next consider an integrated effect of the entire mass-loading region with volume $L \cdot S$ (red area in Fig. 1), where $L$ is the length of the mass-loading region in the $-x$ direction (the location slides anti-sunward along the escaping ion trajectory), as illustrated in Fig. 1. In this case we should replace $\mathrm{d} \rho / \rho$ in Eq. (3) by the ratio of the total mass fluxes into a box of volume $S(x) \mathrm{d} x$, i.e. $m_{\text {load }} f_{\text {load }}^{\prime}(x) \mathrm{d} x$ for mass loading ions and $\rho(x) u(x) S(x)$ for the solar wind ions, where $f_{\text {load }}^{\prime}(x) \mathrm{d} x$ is the $\mathrm{O}^{+}$outflow rate flowing into $S(x) \mathrm{d} x$ at $x$ from the lower boundary. By integrating this over $\mathrm{d} x$, we have

$$
\begin{aligned}
\frac{\Delta K}{K_{\text {in }}} & =-\frac{1}{2} \int^{L} \frac{m_{\text {load }} f_{\text {load }}^{\prime}(x) \mathrm{d} x}{\rho(x) u(x) S(x)}, \\
& =-\frac{m_{\text {load }}}{2 m_{p} n_{\mathrm{sw}} u_{\mathrm{sw}}^{2} S(x=0)} \int^{L} f_{\text {load }}^{\prime}(x) u(x) \mathrm{d} x,
\end{aligned}
$$

where we have used momentum conservation of $\rho(x) u^{2}(x) S(x) \quad$ total force of the solar wind over $S(x)=$ const).

Here, we assume low mixing ratio of mass-loading ions in the solar wind flow such that changes of $u(x)$ and $f_{\text {load }}^{\prime}(x)$ along $x$ are not large over distance $L$. This assumption is reasonable for the observed very low mixing ratio ( $\sim 1 \%$ level). In such a case, the integral part in Eq. (5) (which, strictly speaking, depends on the deceleration profile, and hence on $n_{\mathrm{sw}}$ and $\left.S(x=0)\right)$ does not depend much on these parameters. Therefore, by inserting $K_{\text {in }}$ from Eq. (4), we finally have

$$
\begin{aligned}
\Delta K & =-\frac{1}{4} m_{\text {load }} u_{\mathrm{sw}} \int^{L} f_{\text {load }}^{\prime}(x) u(x) \mathrm{d} x \\
& \sim-\frac{1}{4} m_{\text {load }} F_{\text {load }} u_{\mathrm{sw}}^{2},
\end{aligned}
$$

where $F_{\text {load }}=\int^{L} f_{\text {load }}^{\prime}(x) \mathrm{d} x$ is the total escaping flux into the mass-loading region. Both the solar wind density $n_{\mathrm{sw}}$ and the cross section of the solar wind access $(S)$ disappear from the expression of $\Delta K$ in Eq. (5), because a larger $S$ or a higher 
$n_{\text {sw }}$ means more incident kinetic power but at the same time a smaller conversion rate due to a lower mass mixing rate.

According to Eq. (5), a $u_{\mathrm{sw}}=200 \mathrm{~km} \mathrm{~s}^{-1}$ solar wind inflow just before the mass-loading region and a $\mathrm{O}^{+}$escape rate of $F_{\text {load }}=1-2 \times 10^{25} \mathrm{~s}^{-1}$ into the mass-loading region predict $\Delta K=0.3-0.5 \times 10^{10} \mathrm{~W}$, narrowing the above estimate. This value is comparable to the power that the cusp current carries (up to a total of $10^{6} \mathrm{~A}$ over a $10^{4} \mathrm{~V}$ potential drop for relatively strong IMF; Yamauchi and Araki, 1989), and hence the kinetic energy extracted by the mass loading is indeed a good candidate for its supplier.

In this estimate, the kinetic energy of the bulk flow in the height $(z)$ direction is ignored. In reality, the effect of the anti-sunward bending of the geomagnetic field converts the kinetic energy of the escaping $\mathrm{O}^{+}$from the $z$ direction to the $-x$ direction by the centrifugal acceleration, and the mirror effect of the dipole-like spread of the magnetic field converts the $\mathrm{O}^{+}$gyromotion to the $-x$ direction (e.g. Alfvén and Fälthammar, 1963, Sect. 2.3.6). These additional contributions to momentum to the escaping ions in the $-x$ direction reduce the amount of the kinetic energy conversion from the solar wind to the escaping ions. However, the strongest escape is mainly found in the dayside rather than in the nightside polar cap (e.g. Peterson et al., 2008; Nilsson et al., 2012; Slapak et al., 2017), and this effect should not significantly reduce the energy extraction rate $\Delta K$.

\subsection{Destination of the converted energy}

The main destination of the converted energy cannot be heating (cycloid-like gyromotion) of the escaping $\mathrm{O}^{+}$, because they are already heated with comparable gyro-velocity as the decelerated solar wind before entering the mass-loading region, as mentioned above. On the other hand, deceleration of the solar wind means charge separation in the azimuthal $(\eta)$ and longitudinal $(\xi)$ directions with respect to the local flow direction (see Fig. 3 for directions) due to finite inertia (finite gyroradius) effects (Willis, 1975; Yamauchi et al., 1993a) because the direction of the azimuthal charge separation is opposite to the motional electric field, as illustrated in Fig. 3 (Willis, 1975; Yamauchi and Slapak, 2017). Thus, electrostatic energy accumulates during this process. One example of charge separation by the solar wind deceleration is the bow shock, where the electric current is expected to flow in the dynamo direction, i.e. against the motional electric field of the solar wind (e.g. Tanaka, 1995).

Meanwhile, the azimuthal deflection also adds extra gyromotion to the $\mathrm{H}^{+}$flow, i.e. additional non-laminar velocity to the $\mathrm{H}^{+}$motion, increasing the ion temperature. Distribution of the extracted kinetic energy to different destinations, i.e. to the dynamo current system (charge separation; see Fig. 3) and to thermal energy (extra gyration), depends on the degree of the solar wind deceleration (degree of the compression over a limited region in space), with sharper compression (e.g. under high Mach number) leading to a larger frac-

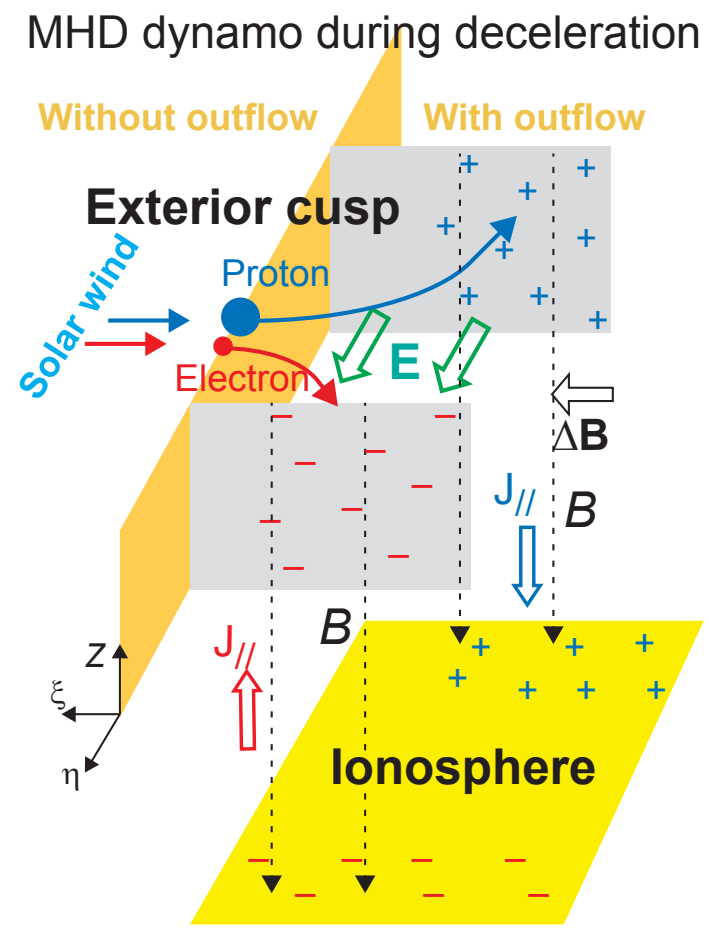

Figure 3. Illustration of azimuthal charge separation in a constant magnetic field $B$, when the solar wind inflow is decelerated by the start of mass loading with the outflowing ionospheric ions (the boundary of the start of the outflowing region is shown by an orange plane). The coordinates $\xi$ and $\eta$ are defined by the flow direction at this boundary, and $\xi$ is not necessarily aligned with the solar wind direction $(-X)$ outside the magnetosphere when IMF points dawnward or duskward. The positive (negative) charges are always deflected opposite to (along) the motional electric field $\boldsymbol{E}$ of the flow, resulting in a dynamo current that causes a magnetic deviation $(\Delta \boldsymbol{B})$. Because of the ionosphere, where charges can move along the electric field across the magnetic field, the accumulated charges form a current circuit through the field-aligned currents $(J / /$ with blue and red arrows giving directions along $\boldsymbol{B}$ ) closing in the ionosphere.

tion converting into thermal energy. This applies to the exterior cusp: the pressure increase (solar wind deceleration) by mass-loading effect should be enhanced in the narrowed cusp geometry, and might even become a shock-like structure (Yamauchi and Lundin, 1997). However, the location of the mass loading is not limited to the exterior cusp, but is extended in both the azimuthal and flow directions. The plasma mantle is in fact the mass loading area, as illustrated in Fig. 1. Therefore, we ignore such kind of mixing effect in the following formulation. Without a shock formation that converts a large part of the kinetic energy into thermal energy, a substantial amount of the kinetic energy will be converted to electrostatic potential energy.

Unlike the bow shock, the region of compression by mass loading is electromagnetically connected to the ionosphere through relatively straight geomagnetic fields (cur- 
vature might cause non-aligned currents, and we ignore it here), where Pedersen currents in the electric field direction flow perpendicular to the geomagnetic field. Then, the electric current (separated charges) relevant to the mass loading will be consumed by a current circuit that closes in the ionosphere. In such a case, a large fraction of the original kinetic energy is expected to be converted to electrostatic energy and to the current system.

\section{Kp dependence and IMF dependence}

\subsection{Kp dependence for $\mathrm{Kp} \leq 7$}

Next we consider the Kp dependence. Slapak et al. (2017) recently showed a clear $\mathrm{Kp}$ dependence of $\mathrm{O}^{+}$outflow rate in the plasma mantle as $\sim 3.9 \times 10^{24} \exp (0.45 \mathrm{Kp}) \mathrm{s}^{-1}$ for $\mathrm{Kp}=1-7$, from $0.5 \times 10^{25} \mathrm{~s}^{-1}$ for $\mathrm{Kp}=1$ to $10 \times 10^{25} \mathrm{~s}^{-1}$ for $\mathrm{Kp}=7$, with a 1-2 orders of magnitude range for a given geomagnetic activity condition (note that $\mathrm{O}^{+}$in the magnetosheath is about one-third of this value). Applying this relation to Eq. (5), we have a proportionality relation

$\Delta K \propto u_{\mathrm{sw}}^{2} \exp (0.45 \mathrm{Kp})$

To obtain the Kp dependence of the energy conversion, we need to know the $\mathrm{Kp}$ dependence of the solar wind velocity. Newell et al. $(2007,2008)$ derived a best empirical prediction of $\mathrm{Kp}$ from solar wind parameters only, and found that any $\mathrm{Kp}$ dependence primarily represents the solar wind velocity $\left(u_{0}\right)$ dependence with some contribution from IMF and a very small contribution from the solar wind density $n_{0}$ $\left(u_{0}\right.$ and $n_{0}$ are defined upstream of the bow shock, whereas $u_{\mathrm{sw}}$ and $n_{\mathrm{sw}}$ are defined just before entering exterior cusp, because we are interested in $\Delta K$ only in the mass-loading region).

Therefore, a simple empirical relation between $u_{0}\left(\propto u_{\mathrm{sw}}\right)$ and $\mathrm{Kp}$ is enough for the present purpose. Figure $4 \mathrm{a}$ shows the average relation using 50 years of OMNI hourly values since 1965 , which is reduced to $u_{0}=135 \cdot(\mathrm{Kp}+1.2)$. This relation also traces the peak percentage in the occurrence frequency plot of Kp for given $u_{0}$ by Elliott et al. (2013, Fig. 3b). Using this linear relation, Eq. (6) becomes

$$
\Delta K \propto(\mathrm{Kp}+1.2)^{2} \exp (0.45 \mathrm{Kp})
$$

or

$\log _{10}(\Delta K)=0.2 \cdot \mathrm{Kp}+2 \cdot \log _{10}(\mathrm{Kp}+1.2)+$ constant.

Equation (7) predicts a nearly linear relationship between $\log _{10}(\Delta K)$ and $\mathrm{Kp}$ as shown in Fig. 4b. This linearity comes from the $F_{\text {load }}$ term in Eq. (5), i.e. positive feedback between the increase of ion escaping rate $F_{\text {load }}$ through the increased energy consumption in the ionosphere for high $\mathrm{Kp}$, and extraction of more kinetic energy $\Delta K$ from the solar wind to the current system by the increased $F_{\text {load }}$.

\subsection{Kp dependence for $\mathrm{Kp}>7$}

For the relationship given above, we expect additional nonlinear effects, mainly for very high $\mathrm{Kp}$, for two reasons: one is the extremely high escape rate observed by the Cluster satellites during $\mathrm{Kp} \geq 8+$, which was much higher than the expected values from the empirical $F_{\text {load }}-\mathrm{Kp}$ relation (Schillings et al., 2017). The resultant $\Delta K$ for these "extreme events" should therefore be higher than the predicted value from Eq. (7), as hand-drawn in Fig. 4b with the red dashed line. Schillings et al. (2017) found such an effect for $\mathrm{Kp} \geq 8+$, while future studies will be needed to establish the geomagnetic activity level (including $\mathrm{Kp}$ ) when such a nonlinearity appears. The second possible factor to add a nonlinear effect in Eq. (7) is the significant enhancement of ion outflow during periods when the solar extreme ultraviolet (EUV) flux is enhanced (Cully et al., 2003; Peterson et al., 2006). This additional effect activates the nonlinear feedback between the ion escaping rate $F_{\text {load }}$ and energy extraction rate $\Delta K$. This would cause much higher geomagnetic activities, causing extremely high $F_{\text {load }}$.

Both extremely high $\mathrm{Kp}$ and high EUV flux are particularly important from the viewpoint of ion escape on a geological timescale. According to the result from the Sun-in-Time project (Dorren and Guinan, 1994), in which G-type stars at different stages are compared in order to model the past Sun, the early Sun is believed to have emitted much higher EUV flux, produced faster solar wind, and caused stronger IMF and coronal mass ejections due to a more active dynamo under much faster rotation compared to present day (Ribas et al., 2005; Wood, 2006; Airapetian and Usmanov, 2016). The faster solar wind and stronger IMF made the past nominal conditions equivalent to the major storm conditions in the present time (Krauss et al., 2012), i.e. extremely high Kp. With these effects only, Slapak et al. (2017) assumed $\mathrm{Kp}=10$ to estimate the ion escape rate for the ancient Earth from their empirical result of the ion escape rate as a function of Kp. It is possible to define $\mathrm{Kp}=10$ for such a purpose because $\mathrm{Kp}$ is defined as the logarithmic of the magnetic disturbance.

In addition, high EUV flux in the ancient time should mean a much higher extraction rate from the kinetic energy for the same solar wind and magnetospheric conditions. Therefore, we expect a higher $\mathrm{O}^{+}$escape rate in the ancient time than the empirical estimation and higher total amount of $\mathrm{O}^{+}$escape over the geological history (e.g. 4 billion years) than the estimate by Slapak et al. (2017) or by Krauss et al. (2012), which already reach $5 \times 10^{17} \mathrm{~kg}$, i.e. $40 \%$ of the total oxygen mass in the atmosphere using the empirical estimation (Slapak et al., 2017) or $130 \%$ of the total oxygen mass in the atmosphere using a proxy method (Krauss et al., 2012). Although the reservoir of oxygen molecules is continuously supplied by the evaporation of the oceans, and although the ancient atmosphere contained a large amount of $\mathrm{CO}_{2}$, we should not 

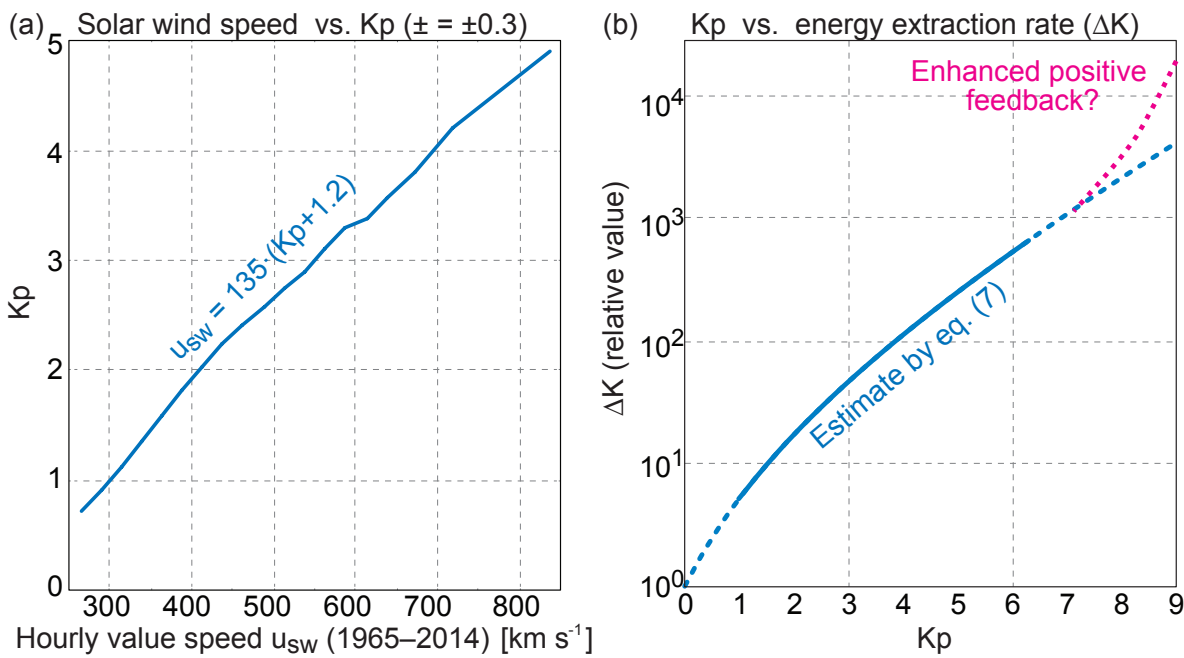

Figure 4. (a) Average relation between $\mathrm{Kp}$ and solar wind velocity $\left(u_{\mathrm{sw}}\right)$ obtained from hourly data (from NASA/OMNI dataset) averaged over 50 years (1965-2014). (b) Predicted increase of the extraction rate of the solar wind kinetic energy $\Delta K$ as a function of Kp compared to $\mathrm{Kp}=0$ given in Eq. (7). Since the $\mathrm{Kp}$-escape relation and the solar wind-Kp relation is reliable only up to $\mathrm{Kp}=5-6$, the estimation for $\mathrm{Kp}>6$ is given in the dashed line, where we have ignored the effect of the nonlinearly enhanced ion flux for extreme high $\mathrm{Kp}(\geq 8+)$ as reported by Schillings et al. (2017). The red dashed line illustrates possible enhancement after adding such an effect for extreme high Kp.

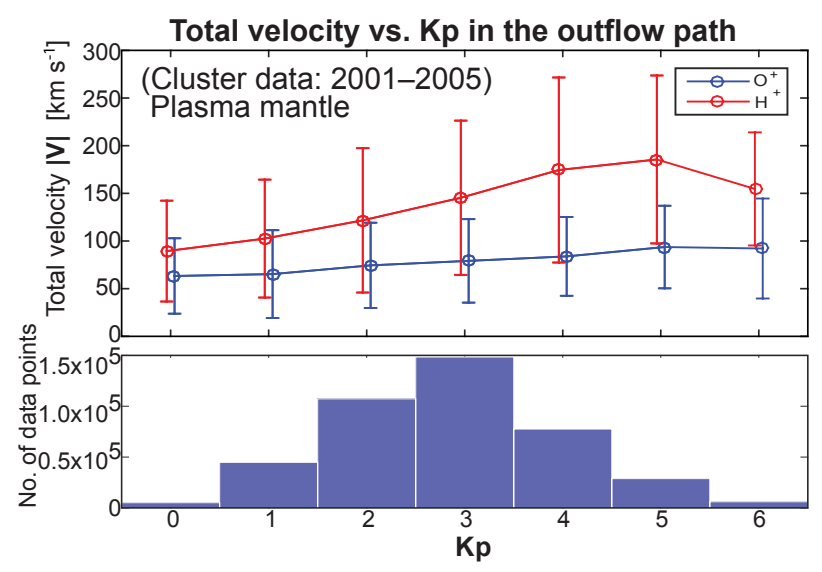

Figure 5. Kp dependence of velocities of $\mathrm{H}^{+}$and $\mathrm{O}^{+}$in the plasma mantle using the same dataset as Fig. 2.

ignore the ion escape when considering atmospheric evolution.

\subsection{IMF dependence}

The remaining major task on this mass-loading dynamo model is the examination of the dependence on different IMF clock angles defined as $\theta_{\mathrm{c}}=\operatorname{atan}\left(B_{Y} / B_{Z}\right)$, where $B_{Y}$ is the duskward component and $B_{Z}$ is the northward component. The prediction of the distribution of separated charges (i.e. distribution of the FACs) must qualitatively agree with observations. Here, in addition to the azimuthal charge separation of the solar wind by the mass-loading deceleration as depicted in Fig. 3, we also include the modification of the solar wind inflow in the exterior cusp by $\theta_{\mathrm{c}}$.

According to the observations (for review, Potemra, 1994; Yamauchi and Slapak, 2017), the northern cusp (injection region of the solar wind) shifts toward postnoon for the IMF $B_{Y}>0$ and prenoon for the IMF $B_{Y}<0$. Also, the solar wind flow in the northern cusp is deflected dawnward for the IMF $B_{Y}>0$ and duskward for $B_{Y}<0$, respectively, flowing toward the noon-midnight meridian. The directions of the shift and deflection are reversed in the Southern Hemisphere for both the cusp location and the solar wind flow. The shift of the cusp location has been successfully modelled by the anti-parallel merging model that predicts the region of minimum magnetic field strength (Crooker, 1979). Also, both the pressure consideration (Yamauchi and Lundin, 1997) and magnetic tension force consideration (e.g. Cowley et al., 1991) predict the deflection direction of the solar wind in the exterior cusp.

We now include the effect of mass loading as illustrated in Fig. 3. When we apply charge separation as illustrated in Fig. 3, we must align the flow direction and the $-\xi$ direction. Therefore, the $-\xi$ direction is no longer aligned with the solar wind direction $(-X)$ outside the magnetosphere. With this modification, the predicted directions of the charge separation in the Northern Hemisphere are illustrated in Fig. 6.

For purely southward IMF $\left(\theta_{\mathrm{c}}=180^{\circ}, B_{Y}=0\right)$, the massloading dynamo will separate solar wind protons $\left(\mathrm{P}^{+}\right)$and electrons $\left(\mathrm{e}^{-}\right)$in the $Y$ direction is illustrated in Fig. 6a in both hemispheres, which is consistent with the flowing direction of the cusp Region 1 currents in both hemispheres (Ohtani et al., 1995; Yamauchi and Slapak, 2017). To con- 

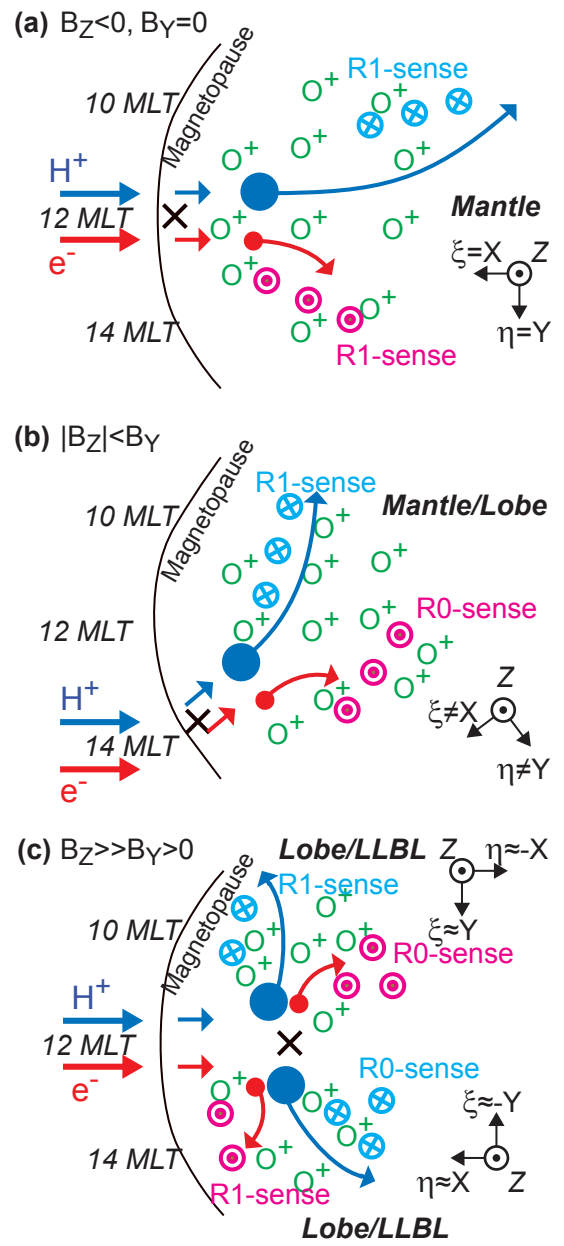

Figure 6. Expected distribution of the positive/negative charges (FAC into/out from the ionosphere) in the Northern Hemisphere in the exterior cusp-plasma mantle region for three different IMF orientations: (a) purely southward IMF, (b) duskward IMF, and (c) strongly northward IMF. Here, both the mass-loading effect and the IMF effect on the solar wind flow in the exterior cusp are considered. Morphologically, the downstream (plasma mantle for southward IMF and LLBL northward IMF) corresponds to the lobe region; see text for further explanations. Longitudinal charge separation (Yamauchi et al., 1993a) is not included here. Blue colour represents flow and charge separation of positive charges $\left(\mathrm{H}^{+}\right)$of the solar wind, and resultant FACs (flowing into the ionosphere). Red colour represents flow and charge separation of negative charges $\left(\mathrm{e}^{-}\right)$of the solar wind, and resultant FACs (flowing out from the ionosphere). Coordinate systems are shown in two ways: $X-Y-Z$ based on the solar wind direction outside the magnetosphere (similar to GSM coordinate), and $\xi-\eta-Z$ based on the local solar wind direction inside the exterior cusp (in the same manner as Fig. 3). The abbreviations "R1-sense" and "R0-sense" indicate polarities of the charge accumulation consistent with the flowing directions of cusp Region 1 FAC and cusp Region 0 FAC, respectively. Note that the different gyroradii between the solar wind protons and electrons are overemphasized in the figure (a $100 \mathrm{~km} \mathrm{~s}^{-1}$ proton in a $30 \mathrm{nT}$ magnetic field has a gyroradius of only $35 \mathrm{~km}$ ). nect the polarities of the separated charges and the FAC direction, we marked these charges as "R1-sense" in the figure ("R0-sense" is defined in the same way).

The mass-loading dynamo makes only one pair of FACs and therefore, does not predict the relatively weak cusp Region 0 FAC in the plasma mantle for purely southward IMF (Iijima et al., 1978; Bythrow et al., 1988). Since the cusp Region 0 FAC for purely southward IMF is relatively weak (e.g. Yamauchi et al., 1993b), this can be explained in different ways - such as the longitudinal charge separation (Yamauchi et al., 1993a) or widening the flow after narrow channel (e.g. Saunders, 1992).

We next consider strongly dawnward or duskward IMF cases $\left(\left|B_{Y}\right|>\left|B_{Z}\right|\right)$. As illustrated in Fig. 6b for duskward IMF $\left(B_{Y}>0\right)$ case, the strong deflection of the solar wind inflow makes the azimuthal ( $\eta$ direction) separation of the charges rotate counter-clockwise, i.e. positive charges toward equatorward of the cusp and negative charges toward poleward of the cusp, respectively, in the Northern Hemisphere, instead of prenoon-postnoon pairs. In the Southern Hemisphere, the pattern rotates clockwise, i.e. negative charges toward the equatorward and positive charges toward the poleward, respectively. As a result, we expect a pair of cusp Region 1 FAC and cusp Region 0 FAC, which is consistent with observations in both hemispheres. The resultant FAC distribution becomes similar to those predicted as a part of the global current system in the simple magnetohydrodynamics (MHD) concept (e.g. Cowley et al., 1991). But as summarized in the Introduction, this single MHD model does not explain the observed independence of the FACs in the cusp and outside the cusp.

Finally, we consider strongly northward IMF cases $\left(B_{Z} \gg\right.$ $\left.\left|B_{Y}\right|\right)$. In such a case, the plasma flow inside the cusp is very slow (Woch and Lundin, 1992; Lavraud et al., 2004) without the plasma mantle (Sckopke et al., 1976; Yamauchi and Lundin, 1993), and therefore we cannot simply apply our model to the cusp flow. However, the solar wind flow may still exist in the magnetosphere, through the prenoon boundary and postnoon of the cusp, flowing toward the low-latitude boundary layer: LLBL (Mitchell et al., 1987; Raeder et al., 1997). In such a situation, we can still apply the mass loading as a combination of strong dawnward IMF and duskward IMF, as illustrated in Fig. 6c, with the entire plasma flow deflected to both sides of the boundary layer. Since we have two different flows towards $+Y$ and $-Y$ directions, we expect two pairs of FACs. The predicted senses of the separated charges are consistent with the observed flowing directions of FACs in both hemispheres (Potemra et al., 1994; Yamauchi and Slapak, 2017, and references therein). However, it is difficult to evaluate whether the observed deceleration is mainly caused by the mass-loading effect, by the magnetic tension force, or by Kelvin-Helmholtz instability. Therefore, the degree of contribution from mass loading during northward IMF is not clear. 


\section{Discussion}

As mentioned in the Introduction, mass-loading deceleration of the solar wind in the open geomagnetic field region in the magnetosphere with the "load" originating in the ionosphere is not a new concept. The new addition in the present study is dividing the mass-loading ions into two independent components. One is the ionospheric (mainly $\mathrm{E}$ region) ions, where the majority of the electrodynamic energy - that is originally coming from the deceleration of the solar wind - is consumed on global scales. The other component corresponds to the escaping ionospheric ions that interact directly with the incoming solar wind in a localized region in the exterior cusp, its vicinity, and the plasma mantle.

These two mass-loading mechanisms are independent of each other in spatial distribution, timescale, and dependence on the solar wind and IMF. The resultant current systems are also independent of each other. Yet, the flowing directions of the current systems by these mechanisms are similar to each other in the cusp region. This is because the present model simply separated the deceleration (by mass loading) and deflection of the solar wind flow (by magnetic tension force) instead of explaining both with the magnetic tension force only.

The estimation of the energy extraction rate $\Delta K$ by the local mass loading associated with the escaping ion (0.3$0.5 \times 10^{10} \mathrm{~W}$ for $200 \mathrm{~km} \mathrm{~s}^{-1}$ solar wind inflow and an $\mathrm{O}^{+}$ escape rate of $1-2 \times 10^{25} \mathrm{~s}^{-1}$ according to Eq. 5) is sufficient to explain the cusp current system, a localized yet large-scale current system that closes with the cusp Region 1 and cusp Region 0 FACs and the ionospheric currents closing these FACs, in the cusp and its vicinity. The predicted location and IMF dependence of this dynamo is also consistent with the cusp Region 1 and cusp Region 0 FACs, which are found to be independent of the dayside Region 1 FACs in the observations (e.g. Ohtani et al., 1995; for review, Yamauchi and Slapak, 2017).

Independent of the mass loading, a compressional structure is predicted near the exterior cusp due to the geometry of the exterior cusp (Yamauchi and Lundin, 1994; Tanaka, 1995). However, the current system due to such compression without the mass-loading effect simply becomes a part of the global current system in the solar wind-magnetosphere interaction. On the other hand, there is a strong seasonal variation (and hence the EUV dependence) of the cusp current system (Fujii and Iijima, 1987; Thomsen, 2004), and this can be a result of combination of the strong EUV dependence of the ion outflow (Cully et al., 2003; Peterson et al., 2008) in the mass-loading model and a geometry effect in the compression model (the exterior cusp is facing the magnetosheath flow in the summer hemisphere). While a statistical study of the EUV (illumination at the ionospheric height) dependence of the $\mathrm{O}^{+}$flow in the exterior cusp and the plasma mantle is needed in the future to diagnose the relative importance of these two mechanisms, if these two mechanisms are coupled, we expect a significant modification of the compressional structure in its location, enhancement of the compression, and enhancement of the Kp dependence (Yamauchi and Lundin, 1997). At the moment it is not possible to say whether such a coupled structure generates the current system independently from that generated by the global solar wind-magnetosphere-ionosphere coupling.

The expected compression (deceleration) of the solar wind also causes a longitudinal charge separation (e.g. Yamauchi et al., 1993a) as well as in the azimuthal direction that is illustrated in Fig. 3. Such a longitudinal charge separation can actually be important according to comet 67P/C-G observations by Rosetta/ICA: the observation showed that the momentum transfer is not limited to the solar wind flow direction, but occurs also in the azimuthal direction (Nilsson et al., 2015; Behar et al., 2016). This indicates that the direction of the charge separation is not necessarily in the azimuthal direction but could largely be in the solar wind direction even without extra factors such as FACs, i.e. as the result of mass loading of newly born ions. The resultant deflection of the solar wind observed by Rosetta/ICA is very often more than $90^{\circ}$ although the speed did not decrease very much when we see the deflected solar wind (Behar et al., 2017; Nilsson et al., 2017). The longitudinal charge separation was also observed at the Earth where FACs flow. Cluster observation found a travelling compressional structure in the inner magnetosphere, which actually caused evening sector aurora, i.e. strong FACs (Yamauchi et al., 2009).

Yamauchi (1994) made a MHD simulation of FACs by such a longitudinal charge separation in a compressional structure, and showed that its intensity is sufficient in explaining the cusp current system with flowing directions consistent with the observations. Since what we showed in the above formulation is that the loss of the kinetic power $(\Delta K)$ is sufficient in powering the cusp current system, the distribution of the energy destination between this type (longitudinal) of current system and the Fig. 3 type (azimuthal) current system is not clear. This problem cannot be solved without numerical simulations that include mass-loading effects.

Such a numerical formulation of the mass-loading effect on the proton flow should be possible even within the MHD regime (and of course with hybrid simulations and the other high-accuracy models) if we violate the conservation of the mass-flux and electric charges, allowing the accumulated charges to flow toward the ionosphere (outside the simulation box). In such a formulation, energy conservation should be case by case because the energy conversion to the thermal energy should be different between a comet case (additional $\mathrm{O}^{+}$is cold) and the Earth's case (additional $\mathrm{O}^{+}$is already heated). 


\section{Conclusions}

By considering the entire region of the mass loading by the escaping ions (exterior cusp and plasma mantle), we estimated the total kinetic energy extraction rate (in power unit) $\Delta K$ from the solar wind inflow in this region. Since the escaping ions are already heated with large gyro-velocity before entering the mass-loading region, $\Delta K$ is converted mainly to other energy forms than thermal energy (through gyromotion), unlike the mass loading of newly ionized atoms of comets and unmagnetized planets. The estimated value of the extracted energy depends mainly on the solar wind velocity accessing to the mass-loading region $\left(u_{\mathrm{sw}}\right)$ and the total mass flux of the escaping ions into the mass-loading region $\left(m_{\text {load }} F_{\text {load }}\right)$, i.e. $\Delta K \sim-m_{\text {load }} F_{\text {load }} u_{\text {sw }}^{2} / 4$, which is about $10^{9-10} \mathrm{~W}$, depending on the solar wind and magnetospheric conditions. This agrees well with the energy of the cusp current system (up to $10^{6} \mathrm{~A}$ over a $10^{4} \mathrm{~V}$ potential drop for a strong-IMF case). Since the IMF $B_{Y}$ dependence can also be explained with this dynamo by considering the $B_{Y^{-}}$ dependent deflection of the solar wind flow in the exterior cusp, the mass-loading dynamo is a good candidate as the source mechanism of the cusp current system.

Using the observed $\mathrm{Kp}$ dependence of the ionospheric ion outflow flux in the mass-loading region, we also estimated the $\mathrm{Kp}$ dependence of the mass-loading dynamo as $\Delta K \propto(\mathrm{Kp}+1.2)^{2} \exp (0.45 \mathrm{Kp})$, or $\log _{10}(\Delta K)=0.2 \cdot \mathrm{Kp}+$ $2 \cdot \log _{10}(\mathrm{Kp}+1.2)+$ constant. Since $F_{\text {load }}$ significantly increases for increased EUV flux, high EUV flux may significantly activate this positive feedback, causing much higher kinetic energy extraction rate by mass loading than the above prediction and hence much higher ion escape rate than the empirical relation. Therefore, the ion escape during the ancient times, when the Sun is believed to have emitted much higher EUV flux than present days, could have been even higher than the currently available highest values based on $\mathrm{Kp}=9$. This raises a possibility that the total $\mathrm{O}^{+}$escape over 4 billion years could have been much larger than the current atmospheric mass, and thus it could have substantially contributed to the evolution of the Earth's atmosphere.

Data availability. The Cluster CODIF and FGM data are provided by Institut de Recherche en Astrophysique et Planetologie in Toulouse, France, and Imperial College London, UK, through the Cluster Science Archive (www.cosmos.esa.int/web/csa). The solar wind OMNI data are provided by NASA and $\mathrm{Kp}$ is provided by GFZ, Adolf-Schmidt-Observatory Niemegk, Germany. Both datasets are taken from NASA/GSFC OMNI site (http://omniweb. gsfc.nasa.gov/ow.html).

Competing interests. The authors declare that they have no conflict of interest.
Acknowledgements. This work is partly supported by the Swedish National Space Board. The Cluster project is performed by the European Space Agency.

The topical editor, Vincent Maget, thanks two anonymous referees for help in evaluating this paper.

\section{References}

Airapetian, V. S. and Usmanov, A.: Reconstructing the Solar Wind from Its Early History to Current Epoch, Astrophys. J. Lett., 817, L24, https://doi.org/10.3847/2041-8205/817/2/L24, 2016.

Akasofu, S.-I.: The solar wind-magnetosphere dynamo and the magnetospheric substorm, Planet. Space Sci., 23, 817-823, https://doi.org/10.1016/0032-0633(75)90018-5, 1975.

Alfvén, H. and Fälthammar, C.G.: Cosmical Electrodynamics, Fundamental Principles, Clarendon, Oxford, 1963.

Axford, W. I. and Hines, C. O.: A unifying theory of high-latitude geophysical phenomena and geomagnetic storms, Can. J. Phys., 39, 1433-1464, https://doi.org/10.1139/p61-172, 1961.

Behar, E., Lindkvist, J., Nilsson, H., Holmström, M., StenbergWieser, G., Ramstad, R., and Götz, C.: Mass-loading of the solar wind at 67P/Churyumov-Gerasimenko: Observations and modelling, Astron. Astrophys. 596, A42, https://doi.org/10.1051/0004-6361/201628797, 2016.

Behar, E., Nilsson, H., Alho, M., Goetz, C., and Tsurutani, B.: The birth and growth of a solar wind cavity around a comet Rosetta observations, Mon. Not. R. Astron. Soc., 469, S396S403, https://doi.org/10.1093/mnras/stx1871, 2017.

Biermann, L., Brosowski, B., and Schmidt, H. U.: The interactions of the solar wind with a comet, Solar Phys., 1, 254-284, https://doi.org/10.1007/BF00150860, 1967.

Breus, T. K., Bauer, S. J., Krymskii, A. M., and Mitnitskii, V. Y.: Mass loading in the solar wind interaction with Venus and Mars, J. Geophys. Res., 94, 2375-2382, https://doi.org/10.1029/JA094iA03p02375, 1989.

Bythrow, P. F., Potemra, T. A., Erlandson, R. E., Zanetti, L. J., and Klumpar, D. M.: Birkeland currents and charged particles in the high-latitude prenoon region: A new interpretation, J. Geophys. Res., 93, 9791-9803, https://doi.org/10.1029/JA093iA09p09791, 1988.

Cladis, J. B.: Parallel acceleration and transport of ions from polar ionosphere to plasmasheet, Geophys. Res. Lett., 13, 893-896, https://doi.org/10.1029/GL013i009p00893, 1986.

Cowley, S. W. H., Morelli, J. P., and Lockwood, M.: Dependence of convective flows and particle precipitation in the high-latitude dayside ionosphere on the $\mathrm{X}$ and $\mathrm{Y}$ components of the interplanetary magnetic field, J. Geophys. Res., 96, 5557-5564, https://doi.org/10.1029/90JA02063, 1991.

Crooker, N. U.: DDayside merging and cusp geometry, J. Geophys. Res., 84, 951-959, https://doi.org/10.1029/JA084iA03p00951, 1979.

Cully, C. M., Donovan, E. F., Yau, A. W., and Arkos, G. G.: Akebono/Suprathermal Mass Spectrometer observations of lowenergy ion outflow: Dependence on magnetic activity and solar wind conditions, J. Geophys. Res.-Space, 108, 1093, https://doi.org/10.1029/2001JA009200, 2003.

Dorren, J. and Guinan, E.: The sun in time: Detecting and modelling magnetic inhomogeneities on solar-type stars, in: 
IAU Coll., 143, The Sun as a Variable Star, edited by: Pap, J. M., Frolich, C., Hudson, H. S., and Solanki, S. K., Cambridge University Press, Cambridge, 206-216, https://doi.org/10.1017/S0252921100024702, 1994.

Dungey, J. W.: Interplanetary magnetic field and the auroral zones, Phys. Rev. Lett., 6, 47, https://doi.org/10.1103/PhysRevLett.6.47, 1961.

Elliott, H. A., Jahn, J.-M., and McComas, D. J.: The Kp index and solar wind speed relationship: Insights for improving space weather forecasts, Space Weather, 11, 339-349, https://doi.org/10.1002/swe.20053, 2013.

Fujii, R. and Iijima, T.: The control of the ionospheric conductivities on large-scale birkeland current intensities under geomagnetic quiet conditions, J. Geophys. Res., 92, 4505-4513, https://doi.org/10.1029/JA092iA05p04505, 1987.

Hasegawa, H., Fujimoto, M., Phan, T.-D., Reme, H., Balogh, A., Dunlop, M. W., Hashimoto, C., and TanDokoro, R.: Transport of solar wind into Earth's magnetosphere through rolled-up Kelvin-Helmholtz vortices, Nature, 430, 755-758, https://doi.org/10.1038/nature02799, 2004.

Heikkila, W. J.: Magnetospheric topology of fields and currents, in: Magnetospheric Currents, edited by: Potemra, T. A., AGU, Washington, D.C., 208-222, https://doi.org/10.1029/GM028p0208, 1984.

Iijima, T. and Potemra, T. A.: Field-aligned currents in the dayside cusp observed by Triad, J. Geophys. Res., 81, 5971-5979, https://doi.org/10.1029/JA081i034p05971, 1976.

Iijima, T., Fujii, R., Potemra, T. A., and Saflekos, N. A.: Fieldaligned currents in the south polar cusp and their relationship to the Interplanetary magnetic field, J. Geophys. Res., 83, 55955603, https://doi.org/10.1029/JA083iA12p05595, 1978.

Kömle, N. I. and Lichtenegger, H. I. M.: Numerical model of timedependent hydrodynamic flows with mass loading, Adv. Space Res., 4, 249-252, https://doi.org/10.1016/0273-1177(84)90036X, 1984.

Krauss, S., Fichtinger, B., Lammer, H., Hausleitner, W., Kulikov, Yu. N., Ribas, I., Shematovich, V. I., Bisikalo, D., Lichtenegger, H. I. M., Zaqarashvili, T. V., Khodachenko, M. L., and Hanslmeier, A.: Solar flares as proxy for the young Sun: satellite observed thermosphere response to an X17.2 flare of Earth's upper atmosphere, Ann. Geophys., 30, 1129-1141, https://doi.org/10.5194/angeo-30-1129-2012, 2012.

Lavraud, B., Phan, T. D., Dunlop, M. W., Taylor, M. G. G. G. T., Cargill, P. J., Bosqued, J.-M., Dandouras, I., Rème, H., Sauvaud, J.-A., Escoubet, C. P., Balogh, A., and Fazakerley, A.: The exterior cusp and its boundary with the magnetosheath: Cluster multi-event analysis, Ann. Geophys., 22, 3039-3054, https://doi.org/10.5194/angeo-22-3039-2004, 2004.

Liao, J., Kistler, L. M., Mouikis, C. G., Klecker, B., and Dandouras, I.: Acceleration of $\mathrm{O}^{+}$from the cusp to the plasma sheet, J. Geophys. Res., 120, 1022-1034, https://doi.org/10.1002/2014JA020341, 2015.

Mitchell, D. G., Kutchko, F., Williams, D. J., Eastman, T. E., Frank, L. A., and Russell, C. T.: An extended study of the low-latitude boundary layer on the dawn and dusk flanks of the magnetosphere, J. Geophys. Res., 92, 7394-7404, https://doi.org/10.1029/JA092iA07p07394, 1987.

Newell, P. T., Sotirelis, T., Liou, K., Meng, C.-I., and Rich, F. J.: A nearly universal solar wind-magnetosphere coupling function in- ferred from 10 magnetospheric state variables, J. Geophys. Res., 112, A01206, https://doi.org/10.1029/2006JA012015, 2007.

Newell, P. T., Sotirelis, T., Liou, K., and Rich, F. J.: Pairs of solar wind-magnetosphere coupling functions: Combining a merging term with a viscous term works best, J. Geophys. Res., 113, A04218, https://doi.org/10.1029/2007JA012825, 2008.

Nilsson, H.: Heavy ion energization, transport, and loss in the Earth's magnetosphere, in: The Dynamic Magnetosphere, edited by: Liu, W. and Fujimoto, M., IAGA, Springer, https://doi.org/10.1007/978-94-007-0501-2_17, 2011.

Nilsson, H., Waara, M., Arvelius, S., Marghitu, O., Bouhram, M., Hobara, Y., Yamauchi, M., Lundin, R., Rème, H., Sauvaud, J.-A., Dandouras, I., Balogh, A., Kistler, L. M., Klecker, B., Carlson, C. W., Bavassano-Cattaneo, M. B., and Korth, A.: Characteristics of high altitude oxygen ion energization and outflow as observed by Cluster: a statistical study, Ann. Geophys., 24, 1099-1112, https://doi.org/10.5194/angeo-24-1099-2006, 2006.

Nilsson, H., Waara, M., Marghitu, O., Yamauchi, M., Lundin, R., Rème, H., Sauvaud, J.-A., Dandouras, I., Lucek, E., Kistler, L. M., Klecker, B., Carlson, C. W., Bavassano-Cattaneo, M. B., and Korth, A.: An assessment of the role of the centrifugal acceleration mechanism in high altitude polar cap oxygen ion outflow, Ann. Geophys., 26, 145-157, https://doi.org/10.5194/angeo-26145-2008, 2008.

Nilsson, H., Barghouthi, I. A., Slapak, R., Eriksson, A., and André, M.: Hot and cold ion outflow: spatial distribution of ion heating, J. Geophys. Res., 117, A11201, https://doi.org/10.1029/2012JA017974, 2012.

Nilsson, H., Wieser, G. S., Behar, E., Wedlund, C. S., Kallio, E., Gunell, H., Edberg, N., Eriksson, A. I., Yamauchi, M., Koenders, C., Wieser, M., Lundin, R., Barabash, S., Mandt, K., Burch, J. L., Goldstein, R., Mokashi, P., Carr, C., Cupido, E., Fox, P. T., Szego, K. , Nemeth, Z., Fedorov, A., Sauvaud, J.A., Koskinen, H., Richter, I., Lebreton, J.-P., Henri, P., Volwerk, M., Vallat, C., and Geiger, B.: Evolution of the ion environment of comet 67P/Churyumov-Gerasimenko - Observations between 3.6 and 2.0 AU, Astron. Astrophys., 583, A20, https://doi.org/10.1051/0004-6361/201526142, 2015.

Nilsson, H., Wieser G. S., Behar, E., Gunell, H., Wieser, M., Galand, M., Wedlund, C. S., Alho, M., C. Goetz, C., Yamauchi, M., Henri, P., Odelstad, E., and Vigren, E.: Evolution of the ion environment of comet 67P during the Rosetta mission as seen by RPC-ICA, Mon. Not. R. Astron. Soc., 469, S252-S261, https://doi.org/10.1093/mnras/stx1491, 2017.

Norqvist, P., André, M., Eliasson, L., Eriksson, A. I., Blomberg, L., Lühr, H., and Clemmons, J. H.: Ion cyclotron heating in the dayside magnetosphere, J. Geophys. Res., 101, 13179-13193, https://doi.org/10.1029/95JA03596, 1996.

Ohtani, S., Potemra, T. A., Newell, P. T., Zanetti, L. J., Iijima, T., Watanabe, M., Yamauchi, M., Elphinstone, R. D., de la Beaujardiere, O., and Blomberg, L. G.: Simultaneous prenoon and postnoon observations of three field-aligned current systems from Viking and DMSP-F7, J. Geophys. Res., 100, 119-136, https://doi.org/10.1029/94JA02073, 1995.

Peterson, W. K., Collin, H. L., Lennartsson, O. W., and Yau, A. W.: Quiet time solar illumination effects on the fluxes and characteristic energies of ionospheric outflow, J. Geophys. Res., 111, A11S05, https://doi.org/10.1029/2005JA011596, 2006. 
Peterson, W. K., Andersson, L., Callahan, B. C., Collin, H. L., Scudder, J. D., and Yau, A. W.: Solar-minimum quiet time ion energization and outflow in dynamic boundary related coordinates, J. Geophys. Res., 113, A07222, https://doi.org/10.1029/2008JA013059, 2008.

Potemra, T. A.: Sources of large-scale Birkeland currents, in: Physical signatures of magnetospheric boundary layer process, edited by: Holtet, J. A. and Egeland, A., Kluwer Academic Publishers, Dordrecht, the Netherlands, 3-27, 1994.

Raeder, J., Berchem, J., Ashour-Abdalla, M., Franck, L. A., Paterson, W. R., Ackerson, K. L., Kokubun, S., Yamamoto, T., and Slavin, J. A.: Boundary layer formation in the magnetotail: Geotail observations and comparisons with a global MHD simulation, Geophys. Res. Lett., 24, 951-954, https://doi.org/10.1029/97GL00218, 1997.

Ribas, S., Guinan, E. F., Güdel, M., and Audard, M.: Evolution of the solar activity over time and effects on planetary atmospheres. I. High-energy irradiances (1-1700 ̊), Astrophys. J., 622, 680694, https://doi.org/10.1086/427977, 2005.

Roederer, J. G.: Global problems in magnetospheric plasma physics and prospects for their solution, Space. Sci. Rev., 21, 23-71, https://doi.org/10.1007/BF00194998, 1977.

Saunders, M. A.: The morphology of dayside Birkeland currents, J. Atmos Terr. Phys., 54, 457-461, https://doi.org/10.1016/00219169(92)90024-F, 1992.

Schillings, A., Nilsson, H., Slapak, R., Yamauchi, M., and Westerberg, L.-G.: Relative outflow enhancements during major geomagnetic storms - Cluster observations, Ann. Geophys., 35, 1341-1352, https://doi.org/10.5194/angeo-35-1341-2017, 2017.

Sckopke, N., Paschmann, Rosenbauer, G.,H., and Fairfield, D. H.: Influence of the interplanetary magnetic field on the occurrence and thickness of the plasma mantle, J. Geophys. Res., 81, 26872691, https://doi.org/10.1029/JA081i016p02687, 1976.

Slapak, R., Nilsson, H., and Westerberg, L. G.: A statistical study on $\mathrm{O}^{+}$flux in the dayside magnetosheath, Ann. Geophys., 31, 1005-1010, https://doi.org/10.5194/angeo-31-1005-2013, 2013.

Slapak, R., Nilsson, H., Westerberg, L. G., and Larsson, R.: $\mathrm{O}^{+}$transport in the dayside magnetosheath and its dependence on the IMF direction, Ann. Geophys., 33, 301-307, https://doi.org/10.5194/angeo-33-301-2015, 2015.

Slapak, R., Schillings, A., Nilsson, H., Yamauchi, M., Westerberg, L.-G., and Dandouras, I.: Atmospheric loss from the dayside open polar region and its dependence on geomagnetic activity: implications for atmospheric escape on evolutionary timescales, Ann. Geophys., 35, 721-731, https://doi.org/10.5194/angeo-35721-2017, 2017.

Szegö, K., Glassmeier, K. H., Bingham, R., Alexander, B., Fischer, C., Haerendel, Gerhard., Brinca, A., Cravens, T., Dubinin, E., Sauer, K., Fisk, L., Gombosi, T., Schwadron, N., Isenberg, P., Lee, M., Mazelle, C., Möbius, E., Motschmann, U., Shapiro, V. D., Tsurutani, B., and Zank, G.: Physics of mass loaded plasmas, Space Sci. Rev., 94, 429-671, https://doi.org/10.1023/A:1026568530975, 2000.

Tanaka, T.: Generation mechanisms for magnetosphere-ionosphere current systems deduced from a three-dimensional MHD simulation of the solar wind-magnetosphere-ionosphere coupling processes, J. Geophys. Res., 100, 12057-12074, https://doi.org/10.1029/95JA00419, 1995.
Thomsen, M. F.: Why $\mathrm{Kp}$ is such a good measure of magnetospheric convection, Space Weather, 2, S11004, https://doi.org/10.1029/2004SW000089, 2004.

Waara, M., Slapak, R., Nilsson, H., Stenberg, G., André, M., and Barghouthi, I. A.: Statistical evidence for $\mathrm{O}^{+}$energization and outflow caused by wave-particle interaction in the high altitude cusp and mantle, Ann. Geophys., 29, 945-954, https://doi.org/10.5194/angeo-29-945-2011, 2011.

Willis, D. M.: The microstructure of the magnetopause, Geophys. J. Int., 41, 355-389, https://doi.org/10.1111/j.1365246X.1975.tb01621.x, 1975.

Woch, J. and Lundin, R.: Magnetosheath plasma precipitation in the polar cusp and its control by the interplanetary magnetic field, J. Geophys. Res., 97, 1421-1430, https://doi.org/10.1029/91JA02487, 1992.

Wood, B. E.: The solar wind and the Sun in the past, Space Sci. Rev., 126, 3-14, https://doi.org/10.1007/s11214-006-9006-0, 2006.

Yamauchi, M.: Numerical simulation of large-scale fieldaligned current generation from finite-amplitude magnetosonic waves, Geophys. Res. Lett., 21, 851-854, https://doi.org/10.1029/94GL00883, 1994.

Yamauchi, M. and Araki, T.: The interplanetary magnetic field By-dependent field-aligned current in the dayside polar cap under quiet conditions, J. Geophys. Res., 94, 2684-2690, https://doi.org/10.1029/JA094iA03p02684, 1989.

Yamauchi, M. and Lundin, R.: A narrow region of electron beams at the poleward edge of the cusp, J. Geophys. Res., 98, 7585-7591, https://doi.org/10.1029/92JA02775, 1993.

Yamauchi, M. and Lundin, R.: Classification of large-scale and meso-scale ion dispersion patterns observed by Viking over the cusp-mantle region, in: Physical Signatures of Magnetospheric Boundary Layer Process, edited by: Holtet, J. A. and Egeland, A., Kluwer Academic Publishers, Dordrecht, the Netherlands, 99-109, https://doi.org/10.1007/978-94-011-1052-5_7, 1994.

Yamauchi, M. and Lundin, R.: The wave-assisted cusp model: Comparison to low-altitude observations, Phys. Chem. Earth, 22, 729-734, https://doi.org/10.1016/S0079-1946(97)00203-6, 1997.

Yamauchi, M. and Slapak S.: Cusp current system: an energy source view, in: Electric Currents in Geospace and Beyond, edited by: Keilling, A., Marghitu, O., and Wheatland, M., AGU Monograph, accepted, 2017.

Yamauchi, M., Lundin, R., and Lui, A. T. Y.: Vorticity equation for MHD fast waves in geospace environment, J. Geophys. Res., 98, 13523-13528, https://doi.org/10.1029/93JA00638, 1993a.

Yamauchi, M., Lundin, R., and Woch, J.: The interplanetary magnetic field By effects on large-scale field-aligned currents near local noon: Contributions from cusp part and noncusp part, J. Geophys. Res., 98, 5761-5767, https://doi.org/10.1029/92JA02934, 1993b.

Yamauchi, M., Dandouras, I., Daly, P. W., Stenberg, G., Frey, H. U., Lindqvist, P.-A., Ebihara, Y., Nilsson, H., Lundin, R., Rème, H., André, M., Kronberg, E. A., Balogh, A., and Henderson, M.: Magnetospheric solitary structure maintained by $3000 \mathrm{~km} / \mathrm{s}$ ions as a cause of westward moving auroral bulge at 19 MLT, Ann. Geophys., 27, 2947-2969, https://doi.org/10.5194/angeo27-2947-2009, 2009. 